\title{
Prevención y solución de conflictos ambientales con la aplicación del mecanismo dispute board
}

Fecha de recepción : 2021-06-10 • Fecha de aceptación: 2021-08-21 • Fecha de publicación: 2021-09-10

Francisco Javier Peña Jaramillo Universidad de los Hemisferios, Ecuador javispj@hotmail.com

https://orcid.org/0000-0002-3309-079X

\section{Resumen}

El presente trabajo tiene por objetivo identificar y exponer las eventuales ventajas jurídicas, que permite aplicar el mecanismo del dispute boards y cómo aportarían en el sistema ecuatoriano de ser adoptado plenamente, como mecanismo pre-arbitral de prevención de conflictos ambientales.

Este evitará que se presenten conflictos ambientales en proyectos que estén catalogados de acuerdo al Sistema Único de Información Ambiental (SUIA) del Ministerio del Ambiente, que requieran licencia ambiental, que se considera que sus impactos y riesgos ambientales son de medio y alto impacto; al contar con esta herramienta jurídica, las partes actuarán de forma inmediata para solucionar el impacto ambiental generado, evitando el conflicto judicial, en pos del cumplimiento del principio de celeridad como lo detalla la normativa jurídica ecuatoriana.

Palabras clave: dispute boards, pre-arbitral, conflicto ambiental, ambiente, prevención ambiental.

\begin{abstract}
The purpose of this paper is to identify and expose the eventual legal advantages of applying the dispute boards mechanism and how it would contribute to the Ecuadorian system if it is fully adopted as a pre-arbitral mechanism to prevent environmental conflicts.
\end{abstract}


This will prevent environmental conflicts from arising in projects that are catalogued according to the Unified Environmental Information System (SUIA) of the Ministry of the Environment, that require an environmental license and whose environmental impacts and risks are considered to be of medium and high impact; by having this legal tool, the parties will act immediately to solve the environmental impact generated, avoiding the judicial conflict, in order to comply with the principle of celerity as detailed in the Ecuadorian legal regulations.

Keywords: dispute boards, pre-arbitration, environmental conflict, environment, environmental prevention. 


\section{Introducción}

En el Ecuador, desde el año 2008 se incorporaron derechos a la naturaleza, lo que permitió una mayor gestión ambiental, facultando al Ministerio de Ambiente y Agua como el ente de control y regulación del cumplimiento de las normas legales, en el margen de respeto a las garantías y derechos constitucionales.

De esta manera, la Constitución de la República (2008) establece derechos y garantías a la madre naturaleza, y como tal, se desencadenan procedimientos y métodos para evacuar las acciones legales y las medidas cautelares que se puedan ejecutar como prevención o resolución de los conflictos ambientales, coadyuvando a una protección integral de la naturaleza y de las partes en discordancia.

La prevención de conflictos es uno de los pilares fundamentales de toda empresa, ya que el mismo establece cuidar la inversión, optimizar los tiempos, y, sobre todo, cuidar el medio ambiente y la salud de las comunidades o poblaciones en áreas de predominio directo. Esta prevención permite la implantación y correcta ejecución de los proyectos en base a la aplicación de las mejores técnicas y cumplimiento de la normativa ambiental.

Durante muchos años, la población ecuatoriana continuamente recibe noticias de varios eventos que han causado afectaciones a comunidades y al ambiente natural, comprometiendo de forma directa los derechos establecidos para la naturaleza. Eventos como daños en la Refinería de Esmeraldas (Bonilla, 2012), lo que ha provocado derrames e incendios que muchas veces han provocado quemaduras, afecciones respiratorias y de piel, destrucción de viviendas, e incluso la muerte.

Hasta la actualidad, existen pasivos ambientales dejados por la operación de la Compañía Texaco, procesos que han concluido en tribunales internacionales, sin que estas heridas profundas a la naturaleza desaparezcan. De acuerdo al diario digital Mongabay, Periodismo Ambiental Independiente en Latinoamérica, publica el 04 de Mayo del 2020 un artículo que menciona el derrame que se produjo en el Oleoducto de Crudos Pesados (OCP) en septiembre del 2019 en el sitio Santa Rosa, así como el daño que se produjo el 7 de abril de 2020 con la ruptura de los dos oleoductos del Ecuador: el Sistema de Oleoductos Trans Ecuatorianos (SOTE) y el OCP (Basantes, 2020), convirtiéndose en daños graves tanto en la fauna, como en la flora.

Este análisis no culminaría por la serie de eventos y afectaciones a la naturaleza y comunidades asentadas en sus territorios que se presentan de forma cotidiana, las autoridades ambientales y la justicia no han tenido una eficacia para sancionar a los responsables, y más bien queda un sabor amargo, la mayoría de afectaciones no se han llegado a sancionar o las sanciones no han llegado a solucionar los impactos presentados. En algunos casos se ha dado indemnizaciones por los impactos presentados, pero la contaminación permanece presente. Las medidas establecidas por las autoridades no han tenido una connotación integral de resolver los conflictos, desde la remediación, restauración natural e indemnizaciones. 
Los recursos naturales son necesarios para la supervivencia de todos los seres humanos, pero los mismos están siendo explotados a una velocidad que supera su regeneración natural, la sobreexplotación ha llevado a la desaparición de los hábitats, necesarios para la flora y la fauna; suelos degradados y altos índices de contaminación. En este sentido, la humanidad entendió que el acceso a la naturaleza y a la propiedad tiene límites, y la misma debe desarrollarse de forma sustentable, lo que conlleva a conservar las múltiples formas y funciones de la naturaleza.

Frente a este panorama, la justicia tiene grandes retos y posibilidades para actuar de forma eficaz frente a los conflictos ambientales, de acuerdo al marco constitucional, como un referente de vida. Así mismo, lo que se torna imprescindible es la responsabilidad de los actores, desde el promotor, comunidad y autoridades, para que se pueda disfrutar de un ambiente sano y libre de contaminación, lo que conlleva a establecer la responsabilidad de los causantes del daño ambiental y con ellos establecer alternativas de solución de conflictos.

En el Ecuador, los sistemas de solución de conflictos se encuentran enmarcados dentro del área administrativa, civil, como indemnizaciones, y en algunos casos, penales. Según la experiencia de estos entes normativos, no ha solucionado, más bien se han incrementado la conflictividad y la agresión ambiental, ya sea por desconocimiento o por costumbres.

El tema ambiental en instancias judiciales es apenas conocido, además de existir una escasa participación ciudadana de la población en procesos de mediación y consenso, lo que ha conllevado a que estos sistemas tradicionales y convencionales no den respuestas efectivas que dé soluciones de forma real y prácticas a estas afectaciones ambientales que agravan la supervivencia de la humanidad.

En el Ecuador, los conflictos ambientales han deteriorado las condiciones de vida de la población y un profundo quebranto del ambiente natural, la contaminación ambiental ha permanecido sin llegar a una solución efectiva y definitiva, y más bien se han convertido en pasivos ambientales que hasta nuestros días permanecen después de varios años de haberse producido el impacto. La normativa actual y los mecanismos de solución se han quedado cortos para poder prevenir el deterioro ambiental y el posterior conflicto socio-ambiental, o herramientas que sean contractuales, por lo que se hace necesario contar con un mecanismo para prevenir dichos conflictos.

Estos no se han resuelto en el país como es el caso de Texaco (Serrano Narváez, 2013), donde se ha dado una serie de argumentos y figuras legales que no han remediado el pasivo ambiental, si en el país los conflictos ambientales permanecen por años sin dar lugar a una resolución definitiva por parte del infractor. Así mismo se actúa de forma ex post cuando ya se ha presentado el impacto y no existe una herramienta previa, la misma que de forma ágil y técnica actúe de inmediato sobre el impacto presentado, de tal forma que no repercuta en el tiempo y no se transforme en un conflicto socio-ambiental.

Las actividades constructivas, mineras, eléctricas e hidrocarburíferas son áreas proclives a las controversias por las complejas relaciones que existen entre la actividad o proyecto, lo social y lo ambiental. Es por ello, que, ante la necesidad de nuevos mecanismos que respondan eficientemente a esas exigencias, aparecen los dispute boards o juntas de resolución de 
conflictos, como medios que se ajustan a la propia naturaleza de este sector. Llegando a constituir actualmente en muchos países: "un excelente medio de reparación de disputas" (Hernández García, 2014).

Para el Ecuador, el tema tiene especial interés porque estamos experimentando un considerable incremento de obras públicas, actividades mineras y petroleras; entre otros factores, gracias al crecimiento económico que se va dando en los últimos años, lo cual constituye un factor importante para el desarrollo del país. De modo que, dichos conflictos acarrean paralizaciones de la obra y presupuestos adicionales. Lo cual genera sobrecostos al Estado, al contratista, la comunidad; así mismo un deterioro de la naturaleza.

Actualmente, en el Ecuador, el arbitraje viene siendo un excelente mecanismo alternativo de solución de controversias en el campo de la construcción, minera y petrolero, nadie duda sobre su justo reconocimiento, debido fundamentalmente a su celeridad en solucionar las controversias. Sin embargo, en la práctica no ha superado aún aquellas exigencias de tiempo, costo y deterioro ambiental que surgen de la misma naturaleza de la actividad ejecutada.

Los dispute board son parte de un sistema integral de solución de disputas, operan en el momento previo al arbitraje, de allí que se les denomina mecanismo pre-arbitral.

Este mecanismo tiene la finalidad de ser una fase o una etapa pre-judicial, de tal modo que se pueda ventilar el conflicto ambiental, en donde las comunidades, conjuntamente con los responsables de los proyectos, sean responsables y puedan llegar a un convenio o solución del conflicto ambiental. Dentro de los conflictos más habituales que se dan entre las partes y que se pueden corregir mediante el dispute board tenemos los presupuestos comunitarios, buenas prácticas ambientales, contingencias ambientales, descargas de aguas contaminadas, manejo de desechos sólidos, líquidos y gaseosos, destrucción de hábitats, caza de especies silvestres, endemismo, tala de bosques y todo lo que tenga que ver con el manejo, conservación del ambiente, sus relaciones con el ser humano y el proyecto.

En consecuencia, este mecanismo permitirá la solución efectiva de forma inmediata, de ahí que las comunidades legitimarían el mismo al ver los resultados; y al mismo tiempo, lo más importante, las soluciones de conflictos serán con celeridad, inmediatez, reducción de costos operativos, no existirá paralizaciones del proyecto; además, las partes podrán nombrar representantes de su entera confianza para el dispute board, lo que evitará un conflicto posterior.

Su aporte al sistema ecuatoriano, de ser adoptado plenamente como mecanismo pre-arbitral de prevención de conflictos ambientales, en la construcción y operación de proyectos, sería positivo y de gran ayuda para la solución inmediata, considerando que muchos de los conflictos generados no se resuelven, esto por la falta de mecanismos legales y operativos que puedan dar por solucionado el conflicto con agilidad. Este mecanismo evitaría que se presenten conflictos ambientales en las actividades, proyectos u obras que estén catalogados de acuerdo al SUIA del Ministerio del Ambiente que requieran licencia ambiental, que son los que se consideran con impactos y riesgos ambientales, de medio y alto impacto, al contar con esta herramienta, las partes 
actuarían de forma inmediata para solucionar el impacto ambiental, evitando el conflicto conforme a la constitución ecuatoriana.

En consecuencia, el presente trabajo toma como ejemplo países latinoamericanos donde se ha instaurado este mecanismo pre-judicial, cuya realidad no es ajena a la nuestra, y en donde han demostrado resultados eficaces para las partes y comunidades en conflicto.

\section{Metodología}

Se aplicó el método científico, el mismo que permitió establecer relaciones entre los hechos o resultados de la aplicación del dispute boards en otros países y los posibles beneficios que acarrearía en nuestro país. Además, se lo aplicó en la obtención de la información bibliográfica científicamente comprobada como revistas jurídicas, discursos y ensayos jurídicos sobre la temática tratada (Ruiz, 2007).

Conjuntamente con el método inductivo - deductivo, se realizó un proceso de observación, para arribar a juicios de valor, conceptos y premisas jurídicas, que permitieron, una mejor conceptualización del tema en estudio (Dávila Newman, 2006).

Así mismo, se utilizó el método estadístico, a través del cual se representó los datos recogidos mediante la técnica de la encuesta, en valores porcentuales y gráficos estadísticos.

Finalmente, se utilizó el método analítico, con el que se logró realizar un análisis exhaustivo de la temática, guiado por información empírica que permitió arribar a datos científicos rescatados por la investigación bibliográfica y la obtenida con la técnica de recolección de datos utilizados (Lopera et al., 2010).

Por último, se aplicó la técnica investigativa de recolección de datos, fue de acuerdo a un cuestionario previamente elaborado, a través del cual se podrá conocer la opinión o valoración, en este caso de abogados o doctores en derecho, en un número de 20.

\section{Resultados}

Las distintas problemáticas que se presentan a diario en relación al medio ambiente, presentan contraposición de intereses, siendo éstas situaciones de interés pública, siendo en la mayoría de los casos la agudización de los impactos en la naturaleza y poblaciones de su entorno, por lo que es una exigencia social el generar acciones o mecanismos que permitan el tratamiento de estos conflictos a la brevedad posible (Quintana, 2011).

La prevención de conflictos es uno de los pilares fundamentales de toda empresa, ya que el mismo establece cuidar la inversión, optimizar los tiempos, y, sobre todo, cuidar el medio ambiente y la salud de las comunidades o poblaciones en áreas de predominio directo. Esta prevención permite la implantación y correcta ejecución de los proyectos en base a la aplicación de las mejores técnicas y cumplimiento de la normativa ambiental. 
Durante muchos años, la población ecuatoriana continuamente recibe noticias de varios eventos que han causado afectaciones a comunidades y al ambiente natural, comprometiendo de forma directa los derechos establecidos para la naturaleza. Eventos como daños en la Refinería de Esmeraldas, lo que provocó derrames e incendios, que muchas veces han provocado quemaduras, afecciones respiratorias y de piel, destrucción de viviendas e incluso la muerte.

Hasta la actualidad, existen pasivos ambientales dejados por la operación de la compañía Texaco, procesos que han concluido en tribunales internacionales, sin que estas heridas profundas a la naturaleza desaparezcan. El derrame que se produjo en el OCP en septiembre del 2019 en el sitio Santa Rosa, así como el daño que se produjo el 7 de abril de 2020 un derrame provocado por el rompimiento de las tuberías de OCP, SOTE y Poliducto, siendo daños graves tanto en la fauna, como en la flora.

Este análisis no culminaría por la serie de eventos y afectaciones a la naturaleza y comunidades asentadas en sus territorios que se presentan de forma cotidiana, las autoridades ambientales y la justicia no ha tenido una eficacia para sancionar a los responsables y más bien queda un sabor amargo, la mayoría de afectaciones no se han llegado a sancionar o las sanciones no han llegado a solucionar los impactos presentados. En algunos casos se ha dado indemnizaciones por los impactos presentados, pero la contaminación permanece presente. Las medidas establecidas por las autoridades no han tenido una connotación integral de resolver los conflictos, desde la remediación, restauración natural e indemnizaciones.

Los recursos naturales son necesarios para la supervivencia de todos los seres humanos, pero los mismos están siendo explotados a una velocidad que supera su regeneración natural, la sobreexplotación ha llevado a la desaparición de los hábitats necesarios para la flora y la fauna; suelos degradados y altos índices de contaminación.

La humanidad entendió que el acceso a la naturaleza tiene límites y la misma debe desarrollarse forma sustentable, lo que conlleva conservar las múltiples formas y funciones de la naturaleza.

Frente a este panorama, la justicia tiene grandes retos y posibilidades para actuar de forma eficaz frente a los conflictos ambientales, de acuerdo al marco constitucional como un referente de vida. Así mismo, lo que se torna imprescindible es la responsabilidad de las partes y como tal del Estado, de garantizar un ambiente sano y al respeto de los derechos constitucionales, a fin de a establecer la responsabilidad de los causantes del daño ambiental y con ellos establecer alternativas de solución de conflictos.

En el Ecuador, los sistemas de solución de conflictos se hallan enmarcados dentro del área administrativa y civil como indemnizaciones, y en algunos casos, penales. Según la experiencia de estos entes normativos, no se ha solucionado, más bien se ha incrementado la conflictividad y la agresión ambiental, ya sea por desconocimiento o por costumbres.

El tema ambiental, en instancias judiciales, es apenas conocido, y la escasa participación ciudadana de la población en procesos de mediación y consenso, ha conllevado a que estos 
sistemas tradicionales y convencionales no dan respuestas efectivas que de soluciones de forma real y prácticas a estas afectaciones ambientales que agravan la supervivencia de la humanidad.

En el país, los conflictos ambientales han deteriorado las condiciones de vida de la población y un profundo deterioro del ambiente natural, la contaminación ambiental ha permanecido sin llegar a una solución efectiva y definitiva, y más bien se han convertido en pasivos ambientales que hasta nuestros días permanecen después de varios años de haberse producido el impacto. La normativa actual y los mecanismos de solución se han quedado cortos para poder prevenir el deterioro ambiental y el posterior conflicto socio-ambiental, o herramientas que sean contractuales, por lo que se hace necesario contar con un mecanismo para prevenirlos. Así mismo, para la obtención de información veraz, de acuerdo al conocimiento jurídico, se aplicó un cuestionario de 6 preguntas a 20 abogados o profesionales del derecho, ya sea en libre ejercicio profesional, servidor público, servidor judicial, entre otros.

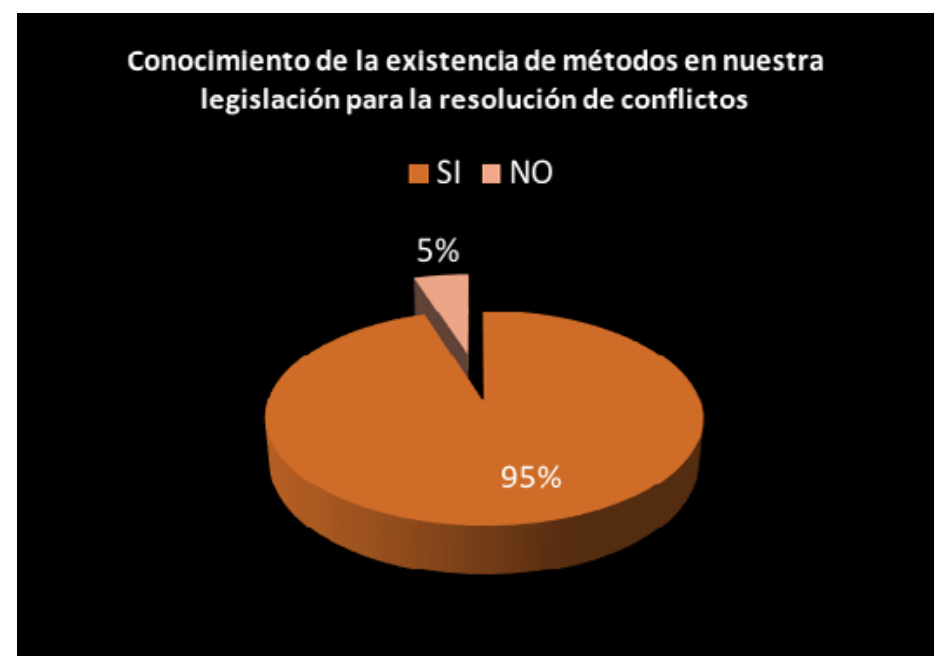

Figura 1. ¿Existen métodos en nuestro sistema jurídico para la resolución de conflictos?

Fuente: elaboración propia

De la población investigada, de acuerdo con la Figura 1, el 95\% menciona que conoce los métodos en nuestro sistema jurídico para la resolución de conflictos, mientras que el $5 \%$ desconoce.

De tal manera que, las personas que menciona tener conocimiento, citan en su mayoría como métodos de solución de conflictos: la vía judicial, la mediación, el arbitraje, los jueces de paz y la conciliación. 


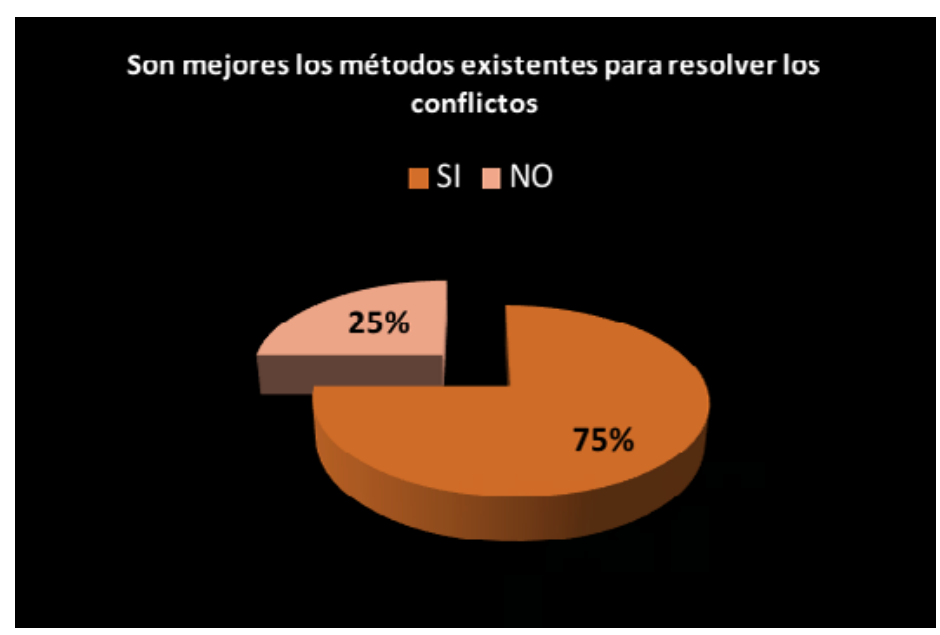

Figura 2. ¿Considera usted que los métodos antes descritos son los mejores para resolver los conflictos? Fuente: elaboración propia

Con respecto a esta interrogante, en la Figura 2 se evidencia que el $75 \%$ de los encuestados consideran que los métodos antes descritos son los mejores para resolver los conflictos, y el $25 \%$ cree que no son los adecuados. Por lo que mencionan que los métodos antes descritos son los mejores para resolver conflictos, por cuanto estos métodos son los adecuados para dar a conocer dos o más partes que manifiestan sus intereses a un tercero neutral, con quien intercambiar ideas y propuestas que permiten resolver sus diferencias. Además de que evitan procesos largos, pero depende de los términos equitativos y no prevalezcan relaciones de poder.

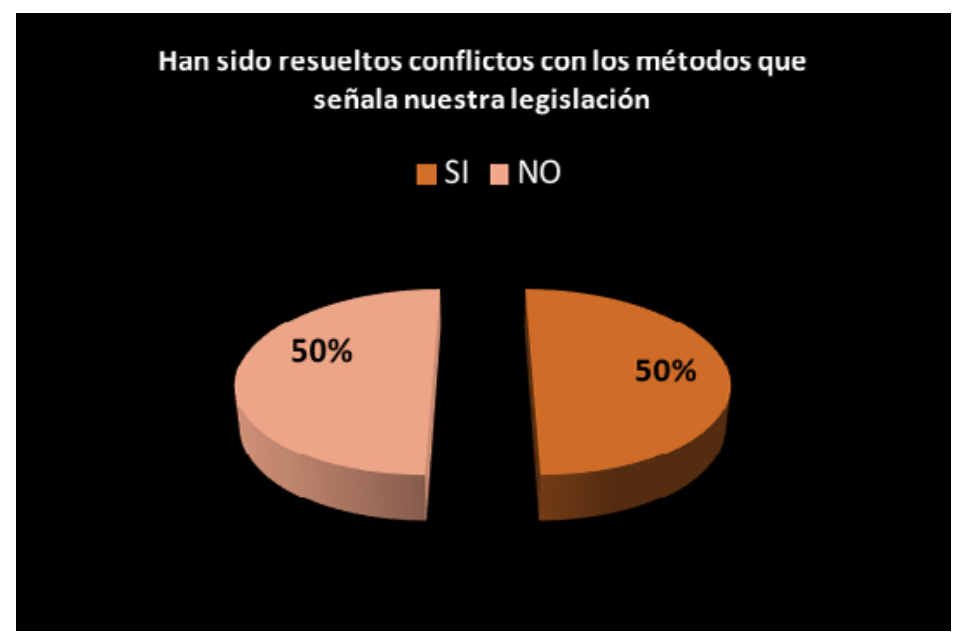

Figura 3. ¿Los conflictos ambientales si han sido resueltos al aplicar los métodos que prevee nuestra Norma?

Fuente: elaboración propia

De la muestra poblacional estudiada, el $50 \%$ opina que los conflictos ambientales si han sido resueltos al aplicar los métodos que prevé esta norma, mientras que el otro $50 \%$ cree que no se han dado solución a muchos conflictos (ver Figura 3). Consecuentemente, el primer grupo considera que el tema ambiental ha sido muy protegido por el país hace 10 años, pero los 
conflictos suscitados han sido solucionados en su gran mayoría y vigilados que se realicen de forma normal y justa para las partes. Así mismo, el segundo grupo opina que, lamentablemente en el Ecuador, este tipo de conflictos son muy comunes, y sería muy importante que se aplicaran métodos alternativos, en este caso el dispute board.

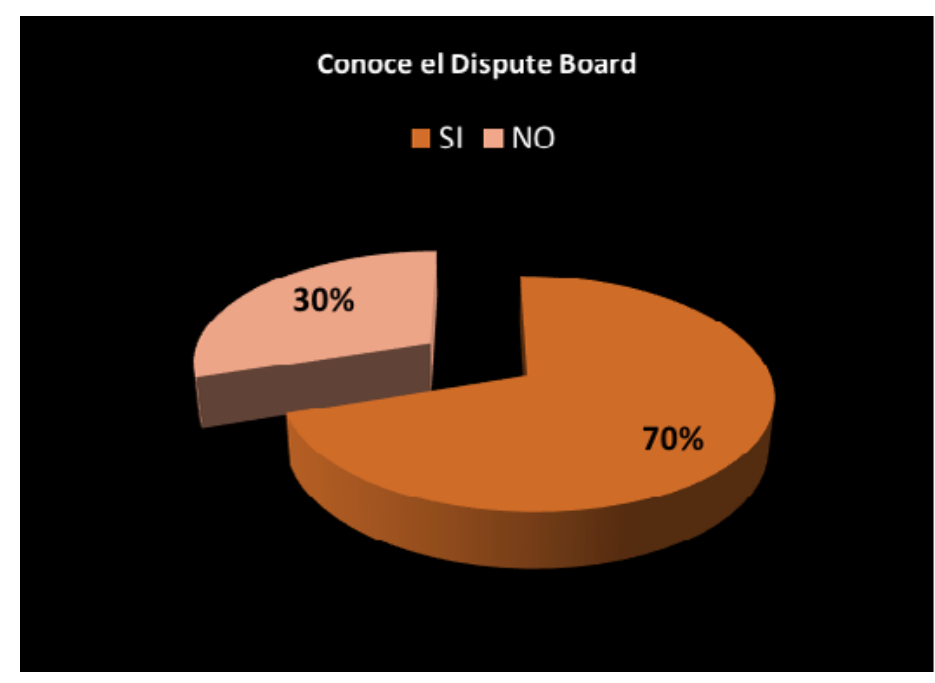

Figura 4. ¿Conoce usted el Dispute Board, como método efectivo para prevenir y resolver conflictos?

Fuente: elaboración propia

El 70\% de los encuestados tienen pleno conocimiento del dispute board (Figura 4), como método efectivo para prevenir y resolver conflictos, y un 30\% desconoce el tema. Definitivamente, en su mayoría se tiene pleno conocimiento de la temática tratada, considerando que éste método es también llamado junta de disputas, y consiste en un método de naturaleza preventiva; es decir, busca evitar los conflictos judiciales, identificando y discutiendo el problema a lo largo del proyecto. Además, se lo considera como una alternativa de solución de conflictos con una solución pacífica, la misma que se puede hacer efectiva para brindar la seguridad al inicio de la ejecución del contrato o acuerdo.

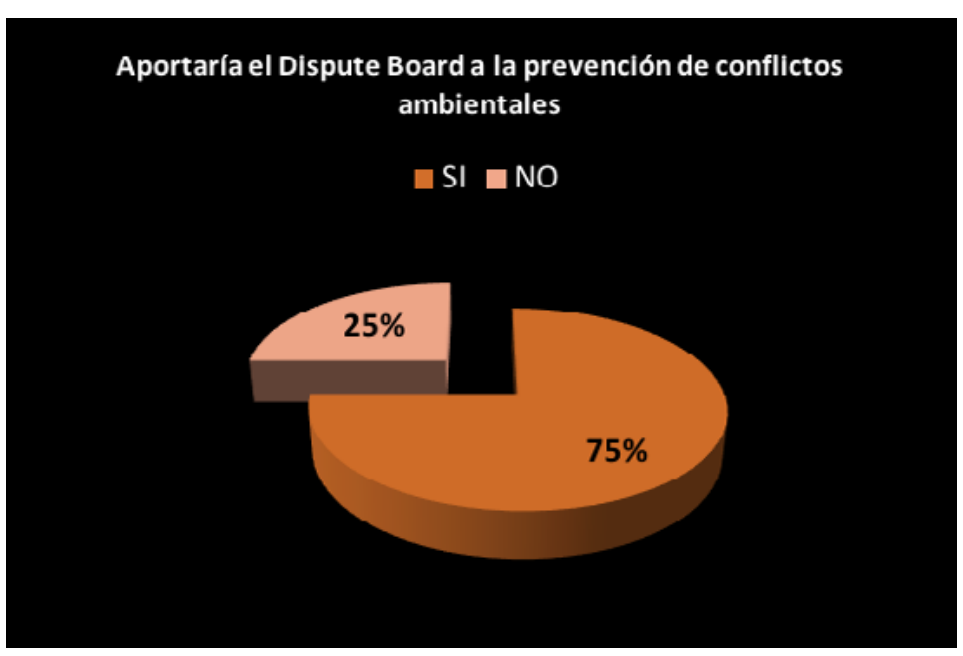


Figura 5. ¿El Dispute Board aportaría a la prevención de conflictos ambientales de acuerdo a lo que establece nuestra constitución?

Fuente: elaboración propia

En relación con la Figura 5, de los 20 encuestados, el 75\% está de acuerdo que el dispute board aportaría a la prevención de conflictos ambientales, de acuerdo a lo que establece la constitución, considerando, además, que un $25 \%$ de la población investigada no está de acuerdo. De tal manera, que, en su mayoría, opinan que se trataría del conflicto de manera integral, evitando llegar a instancias contenciosas, y garantizando los derechos constitucionales, tanto de las partes, como de la naturaleza; pero también se aportaría con la prevención, ya que en la Constitución, en su Art.86, se establece el principio de acción pública, así como también de principios como la celeridad y la eficacia, con los cuales se garantiza un mejor proceder, y como tal, una verdadera justicia.

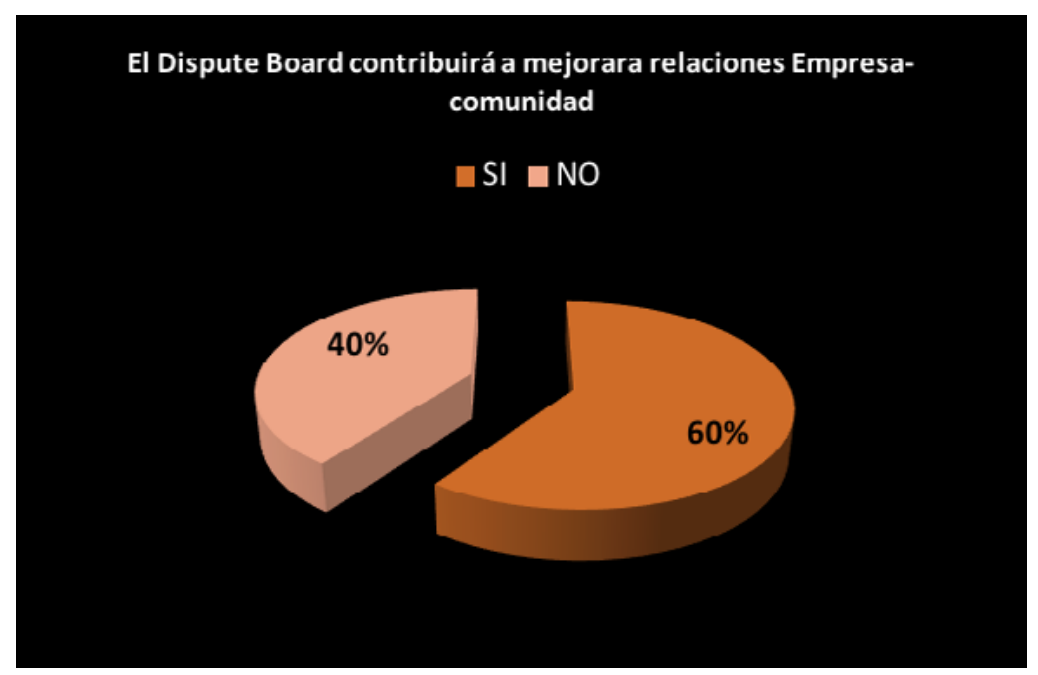

Figura 6. ¿Considera usted que el Dispute Board contribuiría a mejorar las relaciones Empresa- comunidad? Fuente: elaboración propia

El $60 \%$ de los investigados responden que el dispute board contribuiría a mejorar las relaciones empresa-comunidad (ver Figura 6) y el $40 \%$ consideran que no aportaría gran diferencia o cambio en la agilidad de la solución de los conflictos. Por lo que establecen que este método es enfocado a las relaciones dentro de la misma empresa, más no fuera de la misma, tomando en cuenta que la gran mayoría de conflictos ambientales son entre empresa y comunidades, por ser un método preventivo, lo que evitaría litigios legales.

\section{Conclusiones}

Luego de una investigación minuciosa bibliográficamente, así como de forma empírica a través de métodos y técnicas investigativas, se puede arribar a las siguientes conclusiones:

El dispute board es un mecanismo jurídico con resultados importantes en los conflictos aplicados, especialmente de acuerdo a las estadísticas internacionales, ya que dentro de sus mayores 
ventajas está el prevenir conflictos, respetar la equidad de derechos y garantías, la economía judicial y la celeridad de sus resultados (David, 2005).

Este también es un organismo técnico y una herramienta muy importante para la solución de conflictos ambientales, en donde las partes contractuales, de forma rápida y temprana resuelva conflictos frente a un especialista mediante lineamientos claros y legales, ajustados a una realidad actual (Jiménez \& Caivano, 2007).

En el Ecuador, específicamente en los últimos 10 años, se han generado un sinnúmero de conflictos ambientales, ya sea por incumplir la normativa legal o por causar daños en la naturaleza o a las comunidades que habitan en éste entorno, quedando insuficiente la normativa existente para la solución de los conflictos generados.

El mecanismo dispute boards tiene claros principios como son: el impulso de parte, la eventualidad, formalidad y legalidad, la preclusión, la buena fe, el de congruencia, el de inmediación, celeridad, eficacia, entre otros (Escobar, 2014).

En este sentido, es necesario una reforma legal integral donde se complemente el Código del Ambiente y el Código Orgánico Integral Penal, conjuntamente con el respeto estricto a los derechos y garantías que se establecen en la Constitución de la República del Ecuador.

De esta manera, la inclusión de la figura jurídica del dispute board, permitirá grandes beneficios, puesto que se obtendrá mayor agilidad en la solución de conflictos, evitando llegar a litigios largos y engorrosos que dilaten la aplicación de la justicia y el respeto a los derechos de las partes. Serán expertos en temas ambientales con título de tercer nivel con formación formal ambiental y experiencia, quienes integran esta junta que permitirá una solución efectiva.

En la actualidad, es necesario que el Ecuador reforme e incorpore el mecanismo dispute board a la legislación, transformándose en una forma esencial de solución de conflictos ambientales de forma pacífica y de manera integral, por autoridades ajenas a las partes y equitativas en justicia. 


\section{Referencias}

Asamblea Nacional del Ecuador. (2008). Constitución De La República Del Ecuador.

Basantes, A. (04 de mayo de 2020). Ecuador: la rotura del oleoducto OCP revela el impacto de construir en zonas de alto riesgo. MONGABAY. https://es.mongabay.com/2020/05/ecuador-rotura-oleoducto-ocp-pe$\underline{\text { troleo/ }}$

Bonilla, M. (17 de septiembre de 2012). La mayor refinería del país es de alto riesgo. EL COMERCIO. https:// www.elcomercio.com/actualidad/negocios/mayor-refineria-del-pais-de.html

David, J. (2005). Disput resolution, internacional Chamber of Commerce International comercial arbitration. Procedure. Maxwell, (41).

Dávila Newman, G. (2006). El razonamiento inductivo y deductivo dentro del proceso investigativo en ciencias experimentales y sociales. Laurus, 12(Ext), 180-205.

Escobar, A. (2014). Dispute Board como mecanismo alternativo de solución de controversias contractuales en obras de infraestructura. [Tesis de Posgrado, Universidad Andina Simón Bolívar] Repositorio UASB. http:// hdl.handle.net/10644/4183

Hernández García, R. (2014). Dispute boards (paneles de solución de controversias) en Latinoamérica: retos y perspectivas de un fascinante medio de solución de controversias. En Hernández García, R (Coord.), Dispute boards en Latinoamérica: experiencias y retos, Estudio Mario Castillo Freyre, 23, pp. 21-30.

Jiménez Figueres, D., \& Caivano, R. J. (2007). Funcionamiento de los 'dispute boards' de la CCl y su encuadre en el derecho argentino. Revista internacional de arbitraje, 6, 1-19.

Lopera, J., Ramírez, C., Zuluaga, M., \& Ortiz, J. (2010). El método analítico. Revista de Psicología Universidad de Antioquia, 2(4). https://revistas.udea.edu.co/index.php/psicologia/article/view/10294/9594

Ruiz, R. (2007). El método científico y sus etapas.

Quintana Ramírez, A. (2011). El Conflicto Socioambiental y Estrategias de Manejo. Facultad de Ciencias Ambientales de la Universidad Tecnológica de Pereira. (1)

Serrano Narváez, H. (2013). Caso Chevron-Texaco: cuando los pueblos toman la palabra. Serie Magíster. No. 151. UASB-Digital. http://hdl.handle.net/10644/4023 
Copyright (c) 2021 Francisco Javier Peña Jaramillo

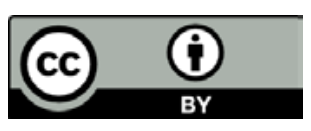

Este texto está protegido bajo una licencia internacional Creative Commons 4.0.

Usted es libre para Compartir-copiar y redistribuir el material en cualquier medio o formato

- y Adaptar el documento - remezclar, transformar y crear a partir del material-para cualquier propósito, incluso para fines comerciales, siempre que cumpla las condiciones de Atribución. Usted debe dar crédito a la obra original de manera adecuada, proporcionar un enlace a la licencia, e indicar si se han realizado cambios. Puede hacerlo en cualquier forma razonable, pero no de forma tal que sugiera que tiene el apoyo del licenciante o lo recibe por el uso que hace de la obra.

Resumen de licencia - Texto completo de la licencia 